\title{
Teniosis y cisticercosis en comerciantes de alimentos en mercados de una área de la ciudad de México
}

\author{
VERÓNICA CRUZ LICEA*, AGUSTÍN PLANCARTE CRESPO**, ISABEL C. MORÁN ÁLVAREZ*, \\ SILVIA VALENCIA ROJAS***, GUSTAVO RODRÍGUEZ SÁNCHEZ**** y LEOPOLDO VEGA FRANCO*
}

\section{TAENIOSIS AND CYSTICERCOSIS IN FOOD TRADERS IN MARKETS IN MEXICO CITY}

In order to know the prevalence of cysticercosis and taeniosis among food traders at the markets of a political delegation in Mexico City, 262 traders were interviewed among 11 markets in Xochimilco. Coproparasitoscopic serial studies and western blot assay in sera were done looking for T. solium eggs and specific antibodies against cysticerci respectively. From interviewed persons, 115 delivered fecal samples and 110 provided blood samples. Prevalence of cysticerco-antibodies was $0,9 \%$ and $\boldsymbol{T}$. solium eggs were not found. Traders used to sell any kind of food related with pig meat, 11,3\% used to breed at home pigs and sacrificed them illegally to sell their meat. Otherwise as observed in other country (Peru), traders in pig meat food that work at markets in Mexico City seldom seem to be exposed to $\boldsymbol{T}$. solium.

Key works: Taenia solium, taeniosis, cysticercosis, food traders, Mexico City.

\section{INTRODUCCIÓN}

La teniosis y la cisticercosis, causadas por la Taenia solium son problemas de salud pública en algunas áreas urbanas y rurales de América Latina, Asia y África. ${ }^{1,2}$ La frecuencia de teniosis en la República Mexicana varía entre $0,1 \%$ y $7,0 \% ;^{3}$ los más afectados son la población entre los 16 y los 45 años de edad. ${ }^{4}$

La Encuesta Nacional Seroepidemiológica (ENSE) de 1987-1988 registró una seroprevalencia de cisticercosis por entidad federativa que varió entre $0,06 \%$ y $2,97 \%$; en el Distrito Federal fue de $2,95 \% .{ }^{5}$ La trascendencia de esta forma invasiva de enfermedad, obedece a que el parásito se aloja en el sistema nervioso central (neurocisticercosis) ${ }^{6}$ por lo que su presencia se expresa con manifestaciones neurológicas entre las que sobresale la epilepsia tardía., 8

Algunas investigaciones epidemiológicas han identificado varios factores asociados a la infección por $T$. solium, como son: bajo nivel socioeconómico, falta de servicios de drenaje y de agua potable, hábitos carentes de cuidados higiénicos personales, consumo frecuente de carne de cerdo, antecedente de expulsión de proglótidos, convulsiones, ataques epilépticos, prácticas domésticas de crianza de cerdos con

\footnotetext{
* Departamento de Salud Pública.

** Departamento de Microbiología y Parasitología, Facultad de Medicina, Universidad Nacional Autónoma de México.

*** Laboratorio de Parasitología y Micología, Instituto Nacional de Pediatría, México.

**** Jurisdicción Sanitaria de Xochimilco, Secretaría de Salud del Distrito Federal, México.

Correspondencia: M en C. Verónica Cruz Licea, Departamento de Salud Pública, Facultad de Medicina, UNAM. Edificio “B”, 6º piso, Ciudad Universitaria. Cp. 04510, México D. F. Tel: 56-23-24-29.

E-mail: veronica_cruz_licea@yahoo.com.mx
} 
acceso de estos animales a heces de humanos..$^{9-13}$

El registro de la frecuencia de cisticercosis en cerdos sacrificados en rastros (establecimiento donde se mata e inspecciona al ganado para su consumo) varía entre $0,004 \%$ y $12,0 \%$; sin embargo, en la población porcina que es sacrificada sin inspección sanitaria la frecuencia de cisticercosis puede ser mayor..$^{14}$ La crianza doméstica de cerdos que se hace frecuentemente en las zonas rurales, es un factor de riesgo importante para la población, debido a que el fecalismo al ras de la tierra facilita que los cerdos consuman las heces de personas infectadas con tenia y, consecuentemente, son sacrificados estos animales sin control sanitario, la carne con cisticercos esta disponible para perpetuar la condición endémica de este parásito. ${ }^{15}$

Las personas dedicadas al comercio de alimentos preparados con carne de cerdo en áreas suburbanas, crían los cerdos o bien los obtienen de rastros clandestinos. En ellos es común observar que el manejo de la carne, o los alimentos preparados con ésta, se hace sin seguir las normas sanitarias: los locales donde expenden estos productos carecen en ocasiones de agua entubada $\mathrm{y}$ drenaje, factores que contribuyen probablemente a que las personas sean expuestas a este parásito al consumir los alimentos preparados con la carne de cerdo. Estas circunstancias son observadas en mercados de las delegaciones políticas de la periferia de la Ciudad de México: Xochimilco es una de ellas. En esta delegación, los comerciantes de alimentos con carne de cerdo, además de comerciar con la carne, crían los cerdos en el patio de su casa, los matan clandestinamente, preparan la carne para su venta e inclusive la consumen. Por eso, el propósito de este trabajo es conocer la frecuencia de infección por $T$. solium. La importancia del estudio radica en que, de estar infectados por tenia quienes manipulan alimentos, aumenta la probabilidad de que ocurra la infección por este parásito entre los consumidores de los productos que expenden.

\section{MATERIAL Y MÉTODOS}

El estudio se llevó a cabo mediante un diseño transversal; se incluyeron todas las personas empadronadas en los locales de alimentos de los mercados establecidos en la Delegación Xochimilco. Éstos se encuentran ubicados en las siguientes localidades: San Andrés Ahuayucan,
San Gregorio Atlapulco, San Luis Tlaxialtemalco, San Mateo Xalpa, Santa Cecilia Tepetlapa, Santa Cruz Alcapixca, Santa María Nativitas, Santiago Tepalcatlalpan, Tulyehualco y en Xochimilco Centro (Tabla 1 y 2). Todos los sujetos incluidos en el estudio habían trabajado no menos de seis meses en el local donde laboraban y aceptaron participar en el estudio, firmando una carta de consentimiento informado donde se describe el propósito del trabajo; todos ellos se comprometieron a que se les tomara una muestra sanguínea y a entregar tres muestras seriadas de materia fecal.

La Delegación Xochimilco, se ubica al sur de la Ciudad de México y representa el 7,9\% de su superficie total; la delegación tiene áreas urbanas $(27,2 \%)$ y rurales $(72,8 \%) \cdot{ }^{16} \mathrm{El}$ Instituto Nacional de Estadística Geográfica e Informática (INEGI) ubica a Xochimilco entre las tres primeras delegaciones productoras de cerdos con más unidades de producción rural de ganado porcino y ocupa el segundo lugar en cuanto al volumen de la producción de carne en canal porcina para el Distrito Federal. ${ }^{17}$

Recolección de la información. La información se obtuvo durante el mes de junio del 2000. Se encuestaron 262 personas (63\% mujeres y $37 \%$ hombres) que laboraban en 167 locales de 11 mercados establecidos en Xochimilco; de estos comerciantes 115 (44\%) entregaron las muestras de materia fecal y 110 (42\%) una muestra de sangre. A todos los que laboraban en locales de alimentos -y aceptaron ser incluidos en el estudiose les aplicó un cuestionario; las preguntas fueron orientadas a obtener información general como: edad, sexo, esco-laridad, e información acerca de su domicilio, (número de habitantes y cuartos de la vivienda, disponibilidad de servicios públicos). También se hicieron preguntas respecto a su higiene personal (lavado de manos antes de comer y después de defecar), e información sobre su salud (con especial interés en los síntomas relacionados a las infecciones estudiadas); por último, se obtuvo información sobre los productos que comercializaban y las características del local comercial (tipo de alimento preparado con carne de cerdo, disponibilidad de los servicios públicos en el local). Luego se les entregaron los recipientes para colectar las muestras de materia fecal y se les instruyó para obtenerlas. Las muestras que colectaron fueron entregadas, por cada persona, en los Centros de Salud de la Secretaría de Salud, 
ubicados cerca de cada mercado; al momento de entregar la muestra de heces, personal de enfermería tomó la muestra de sangre $(5 \mathrm{ml})$ con las medidas de seguridad necesarias.

Técnicas de laboratorio. La materia fecal fue procesada mediante la técnica de centrifugación y sedimentación con formol-éter sugerida por Ritchie. ${ }^{18}$ Se consideró un caso positivo de teniosis cuando se observaran huevos de tenia en el excremento. A los comerciantes con resultado positivo a algún parásito se les proporcionó el tratamiento antiparasitario adecuado por parte del Centro de Salud. Las muestras sanguíneas se centrifugaron y el suero fue analizado con la prueba de EITB ( Enzymelinked Immunoelectrotransfer Blot) para anticuerpos de cisticercos ${ }^{19}$; se consideró un caso positivo de cisticercosis cuando aparecieron una o más de las siete bandas glucoprotéicas específicas en la inmunoelectrotransferencia.

Análisis estadístico. Se calculó la seroprevalencia de cisticercosis y las frecuencias de cada una de las características estudiadas. Los datos de los comerciantes se dividieron para su análisis en dos grupos: en uno se incluyeron los que comerciaban alimentos con carne de cerdo; éstos reciben comúnmente los siguientes nombres: "chicharrón" (piel de cerdo frita), "carnitas" (la carne de cerdo frita en su propia grasa o aceite hirviendo y ordinariamente se consume en tacos), "longaniza" (embutido condimentado con chile, largo y delgado, que se elabora con carne de cerdo), "rellena o moronga" (embutido preparado con sangre de cerdo) y vísceras. El otro grupo de comerciantes vendía, indistintamente, "barbacoa" (carne de borrego cocida en un hoyo bajo tierra), pollo crudo, verduras, frutas, pescados y mariscos. Se usó la prueba de z para medias y la prueba de ji cuadrada $\left(\mathrm{X}^{2}\right)$ para analizar diferencias entre los grupos de comerciantes, el nivel de significancia fue del $95 \%$.

\section{RESULTADOS}

Los factores investigados no mostraron ser diferentes entre los comerciantes que no entregaron muestras biológicas y aquellos que las entregaron (muestras de heces y sanguínea), a excepción de la edad, sexo y la escolaridad: el promedio de edad entre los que entregaron sus muestras biológicas fue de 43,7 $( \pm 14,8)$ años y en los que no lo hicieron fue 35,6 $( \pm 13,6)$ años $(\mathrm{z}=$
4,5; $\mathrm{p}<0,05)$; en cuanto al sexo y la escolaridad, el valor de $X^{2}$ fue de 4,01 y 13,5 (ambos con un valor de $\mathrm{p}<0,05)$.

Entre los individuos que entregaron ambos tipos de muestras (110), cabe resaltar que 45 $(40,9 \%)$ comerciantes tenían un nivel escolar por arriba de los estudios primarios. Algunas de las viviendas de estos comerciantes carecían de servicios públicos: $3,6 \%$ de agua entubada, $10,9 \%$ drenaje, $2,7 \%$ luz eléctrica y $2,7 \%$ tenían piso de tierra. Doce $(10,9 \%)$ comerciantes refirieron defecar al aire libre. En cuanto al hacinamiento (más de dos personas por dormitorio) se presentó en $37,3 \%$ de los comerciantes. En lo que atañe al hábito de lavarse las manos antes de comer y después de defecar, $1,8 \%$ de los comerciantes refirió que no lo hacía; además, $40 \%$ bebían agua sin hervir (Tabla 1).

La seroprevalencia de anticuerpos relacionados con la cisticercosis fue de $0,9 \%$ (1/110). El caso seropositivo fue una mujer de 35 años de edad, de nivel escolar alto (licenciatura), que vivía en hacinamiento, defecaba al aire libre y comía con frecuencia carne de cerdo; no refirió algún síntoma y se dedicaba a la venta de "carnitas", fue referida para estudio.

En el estudio coproparasitoscópico (CPS) practicado a los 115 comerciantes de alimentos que entregaron diariamente, durante tres días, una muestra de materia fecal, no se encontró ninguna tenia. En $58(50,4 \%)$ comerciantes las muestras fueron positivas a alguna otra parasitosis intestinal diferente de T. solium; 38 (33\%) tuvieron infección parasitaria única; en 15 se encontraron dos parásitos $(13 \%)$ y en cinco $(4,4 \%)$ se observaron tres especies. La parasitosis más frecuente fue Blastocystis hominis (41,7\%), seguida por los comensales: Entamoeba coli $(14,8 \%)$, Endolimax nana $(8,7 \%)$ e Iodamoeba bütschlii $(2,6 \%)$. Otras de las especies observadas fueron: Strongyloides stercoralis, Hymenolepis nana, Entamoeba histolytica, Giardia duodenalis y Chilomastix mesnili, todas estas especies con una prevalencia del $1 \%$.

Entre los comerciantes $62(56,4 \%)$ vendían alimentos relacionados con la carne de cerdo y $\operatorname{los} 48(43,6 \%)$ restantes comerciaban con otro tipo de alimentos. Al comparar las características entre ambos grupos, no hubo entre ellos diferencias significativas (Tabla 2).

En cuanto a los comerciantes de alimentos con carne de cerdo (62), $56(90,3 \%)$ refirieron 
Tabla 1. Características de los 262 comerciantes de alimentos de Xochimilco con relación a la entrega de ambas muestras (fecal y sangre), junio del 2000

\begin{tabular}{|c|c|c|c|c|}
\hline \multirow[t]{2}{*}{ Características } & \multicolumn{3}{|c|}{ Entrega de muestras biológicas } & \multirow[b]{2}{*}{$\mathbf{p}$} \\
\hline & $\begin{array}{c}\text { Si }\left(\begin{array}{c}(n=110) \\
n\end{array}\right. \\
\text { nate }\end{array}$ & No $\begin{array}{c}(n=152) \\
n\end{array}$ & $\mathbf{X}^{2}$ & \\
\hline Sexo & & & & \\
\hline Femenino & 77 & 88 & 4,01 & 0,04 \\
\hline Masculino & 33 & 64 & & \\
\hline \multicolumn{5}{|l|}{ Escolaridad baja a } \\
\hline Sí & 65 & 55 & 13,5 & $0,00 *$ \\
\hline No & 45 & 97 & & \\
\hline Vivienda deficiente $^{\mathrm{b}}$ & & & 0,23 & 0,63 \\
\hline Sí & 15 & 24 & & \\
\hline No & 95 & 128 & & \\
\hline Hacinamiento $^{c}$ & & & 3,15 & 0,08 \\
\hline Sí & 41 & 41 & & \\
\hline No & 69 & 111 & & \\
\hline Defecación al aire libre & & & 0,01 & 0,92 \\
\hline Sí & 12 & 16 & & \\
\hline No & 98 & 136 & & \\
\hline Lavado de manos ${ }^{\mathrm{d}}$ & & & 0,53 & 0,47 \\
\hline Sí & 108 & 147 & & \\
\hline No & 2 & 5 & & \\
\hline Consumo de agua sin hervir & & & 0,27 & 0,60 \\
\hline Sí & 44 & 56 & & \\
\hline No & 66 & 96 & & \\
\hline Consumo de carne de cerdo & & & 2,87 & 0,09 \\
\hline Sí & 101 & 129 & & \\
\hline No & 9 & 23 & & \\
\hline -más de tres veces/semana & & & 0,05 & 0,82 \\
\hline Sí & 23 & 30 & & \\
\hline No & 87 & 122 & & \\
\hline Presencia de síntomas ${ }^{\mathrm{e}}$ & & & 1,02 & 0,31 \\
\hline 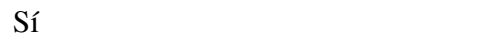 & 36 & 59 & & \\
\hline No & 74 & 93 & & \\
\hline Local comercial deficiente ${ }^{b}$ & & & 0,04 & 0,84 \\
\hline Sí & 28 & 37 & & \\
\hline No & 82 & 115 & & \\
\hline Vende alimentos con carne de cerdo & & & 2,40 & 0,12 \\
\hline Sí & 62 & 100 & & \\
\hline No & 48 & 52 & & \\
\hline Cría doméstica de cerdos & & & 0,30 & 058 \\
\hline Sí & 12 & 20 & & \\
\hline No & 98 & 132 & & \\
\hline Matanza casera ${ }^{f}$ & & & 3,06 & 0,08 \\
\hline Sí & 14 & 32 & & \\
\hline No & 96 & 120 & & \\
\hline
\end{tabular}

" $\mathrm{p}<0,001$

${ }^{a}$ No sabe leer ni escribir o haber cursado hasta estudios primarios

${ }^{\mathrm{b}}$ Falta algún servicio público (agua, drenaje o luz) o tiene piso de tierra

${ }^{\mathrm{c}}$ Más de dos personas por cuarto destinado para dormir

d Antes de comer y después de ir al baño

${ }^{\mathrm{e}}$ Referir algún síntoma: dolor de cabeza, desmayos, movimientos involuntarios en piernas y brazos, defecar proglótidos, epilepsia.

${ }^{\mathrm{f}}$ Procedencia de la carne de cerdo para la venta. 
Tabla 2. Frecuencia de los factores estudiados en los comerciantes de alimentos de mercados de Xochimilco que entregaron sólo muestra de sangre, junio del 2000

\begin{tabular}{|c|c|c|c|c|}
\hline \multirow[t]{3}{*}{ Características } & \multicolumn{2}{|c|}{ Comercio con carne de cerdo } & \multirow{3}{*}{$\mathbf{X}^{2}$} & \multirow{3}{*}{$\mathbf{p}$} \\
\hline & Si $(n=62)$ & No $(n=48)$ & & \\
\hline & $\mathbf{n}$ & & & \\
\hline \multicolumn{5}{|l|}{ Sexo } \\
\hline Femenino & 43 & 34 & \multirow[t]{2}{*}{0,03} & \multirow[t]{2}{*}{0,87} \\
\hline Masculino & 19 & 14 & & \\
\hline Escolaridad baja ${ }^{a}$ & & & \multirow{3}{*}{0,07} & \multirow[t]{3}{*}{0,78} \\
\hline Sí & 39 & 26 & & \\
\hline No & 23 & 22 & & \\
\hline Vivienda deficiente $^{\mathrm{b}}$ & & & \multirow[t]{3}{*}{2,03} & \multirow[t]{3}{*}{0,15} \\
\hline Sí & 11 & 4 & & \\
\hline No & 51 & 44 & & \\
\hline Hacinamiento $^{c}$ & & & \multirow[t]{3}{*}{0,13} & \multirow[t]{3}{*}{0,72} \\
\hline Sí & 24 & 17 & & \\
\hline No & 38 & 31 & & \\
\hline Defecación al aire libre & & & \multirow[t]{3}{*}{0,58} & \multirow[t]{3}{*}{0,45} \\
\hline Sí & 8 & 4 & & \\
\hline No & 54 & 44 & & \\
\hline Consumo de agua sin hervir & & & \multirow[t]{3}{*}{1,21} & \multirow[t]{3}{*}{0,27} \\
\hline Sí & 22 & 22 & & \\
\hline No & 40 & 26 & & \\
\hline Consumo de carne de cerdo & & & \multirow[t]{3}{*}{0,42} & \multirow[t]{3}{*}{0,51} \\
\hline Sí & 56 & 45 & & \\
\hline No & 6 & 3 & & \\
\hline - más de tres veces/semana & & & \multirow[t]{3}{*}{0,86} & \multirow[t]{3}{*}{0,35} \\
\hline Sí & 11 & 12 & & \\
\hline No & 51 & 36 & & \\
\hline Presencia de síntomas ${ }^{d}$ & & & \multirow[t]{3}{*}{1,23} & \multirow[t]{3}{*}{0,27} \\
\hline Sí & 23 & 13 & & \\
\hline No & 39 & 35 & & \\
\hline Local comercial deficiente ${ }^{b}$ & & & \multirow[t]{3}{*}{0,96} & \multirow[t]{3}{*}{0,33} \\
\hline Sí & 18 & 10 & & \\
\hline No & 44 & 38 & & \\
\hline Cría doméstica de cerdos & & & \multirow[t]{3}{*}{0,02} & \multirow[t]{3}{*}{0,88} \\
\hline Sí & 7 & 5 & & \\
\hline No & 55 & 43 & & \\
\hline
\end{tabular}

${ }^{a}$ No sabe leer ni escribir o haber cursado hasta estudios primarios

${ }^{\text {b }}$ Falta algún servicio público (agua, drenaje o luz) o tiene piso de tierra

${ }^{c}$ Más de dos personas por cuarto destinado para dormir

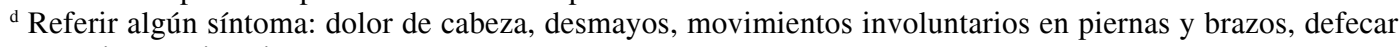
proglótidos, epilepsia.

${ }^{\text {e }}$ Procedencia de la carne de cerdo para la venta.

comer esta carne por lo menos de una a dos veces a la semana y $11(17,7 \%)$ la consumían más de tres veces por semana; de los comerciantes que consumen carne de cerdo $41(73,2 \%)$ refirieron comer de lo que vendían o bien de otros locales comerciales del mismo mercado.

De acuerdo a los síntomas que, alguna vez, habían presentado, los comerciantes que venden alimentos con carne de cerdo mencionaron: cinco con desmayos, 17 con dolores de cabeza frecuentes, 12 con movimientos involuntarios en piernas y brazos, uno con ataques epilépticos y cuatro refirieron haber defecado "tallarines" (como la gente llama a los proglótidos). Se encontró que nueve comerciantes de alimentos relacionados con la carne de cerdo presentaron más de dos síntomas de los cuatro mencionados y en el grupo de comerciantes de otro tipo de alimentos cinco refirieron presentar más de dos síntomas. En cuanto al conocimiento sobre la 
cisticercosis (también conocida entre ellos como: "granizo" o "granillo") y su relación con el cerdo, se encontró que 44 (71\%) de los comerciantes de carne de cerdo, desconocían la enfermedad.

En cuanto a la procedencia de la carne de cerdo $48(77,4 \%)$ dijeron que provenía del rastro y $14(22,6 \%)$ informaron que era de matanza casera. Entre estos últimos, siete comerciantes criaban los cerdos y dos de estos permitían que los cerdos deambularan libres. El resto de los comerciantes refirieron acudir a comprar los animales en criadores de cerdos. Los locales de los mercados en los que se venden los alimentos con carne de cerdo $45(72,6 \%)$ contaban con agua entubada, $44(71,0 \%)$ contaban con drenaje, $50(80,6 \%)$ tenían luz eléctrica y $17(27,4 \%)$ piso de tierra.

\section{DISCUSIÓN}

El hombre adquiere la teniosis por la ingestión de carne de cerdo infectada con cisticercos, mientras que el cerdo y el ser humano adquieren la cisticercosis al consumir alimentos o agua contaminados con excremento humano que contenga huevos de $T$. solium..$^{20}$ La teniosis se debe a que en México existe el consumo de carne de cerdo criado para consumo familiar y sacrificado sin inspección sanitaria; o bien, en el caso de ser inspeccionada, la venta de cerdos con cisticercos con infección leve es permitida, esto queda a juicio del inspector sanitario: a los cerdos se les "cepillan" los cisticercos de los músculos y entonces la carne es destinada para cocción o fritura. ${ }^{21}$ Esta carne de cerdo es regularmente utilizada en la preparación de comida popular tal como "carnitas", "cecina enchilada", "longaniza", "rellena o moronga"; alimentos que en ocasiones son consumidos insuficientemente cocidos, lo que evita la destrucción o muerte del cisticerco. ${ }^{22}$

A veces la venta y consumo de estos alimentos, suele hacerse en la calle, donde se encuentran expuestos a la contaminación por materia fecal $^{23}$; o bien, pueden ser preparados por personas portadoras de la fase adulta de $T$. solium y pueden ocasionar la cisticercosis en los consumidores de estos alimentos; tal como se ha descrito en una comunidad judía ortodoxa de Nueva York ${ }^{23}$, en donde los casos encontrados en la comunidad fueron causados por una trabajadora doméstica que preparaba los alimentos portadora de la tenia.

En el presente estudio, realizado en mercados de Xochimilco, entre los 110 vendedores de alimentos, se encontró $0,9 \%$ de seroprevalencia a cisticercosis y ningún portador de tenia. Estos resultados difieren de los trabajos publicados, como el realizado en Perú entre comerciantes de "chicharrones" 25 , donde se encontró una seroprevalencia de 23,3\% utilizando EITB y una prevalencia de teniosis de $8,6 \%$ utilizando CPS, también mencionan que entre las personas dedicadas a criar de manera doméstica cerdos aumenta la probabilidad de presentar anticuerpos a $T$. solium; y esta probabilidad continúa en aumento si además el comerciante mata los cerdos en su casa, vende la carne cruda o la prepara en "chicharrón". Las diferencias entre los estudios podrían deberse a que en la población de Perú existían factores como: carencia de drenaje en las viviendas (una de cada tres viviendas tiene letrina) y crianza doméstica de cerdos (de uno a tres por familia), factores críticos para el desarrollo del ciclo de vida de T. solium, mientras que los hallazgos del presente estudio, se encontró que la mayoría de los comerciantes de alimentos tienen en sus viviendas y locales servicio de drenaje y agua entubada, la procedencia de la carne de cerdo para la venta en su mayoría proviene del rastro, además mencionan tener adecuados hábitos higiénicos (consumo de agua hervida, lavado de manos antes de comer y después de ir al baño), estos factores evitan o interrumpen el ciclo de vida de la $T$. solium; de aquí, probablemente, la razón de la baja prevalencia encontrada. No obstante, el factor de adecuados hábitos higiénicos es cuestionable ya que la mayoría de los vendedores pudieron haber contestado de manera falsa considerando que era la respuesta correcta.

Por otro lado, se encontraron individuos que presentaron factores considerados como de riesgo para T. solium: hacinamiento, consumo frecuente de carne de cerdo, desconocimiento de la relación entre la tenia en humanos y la cisticercosis en el cerdo (conocida localmente "bolitas blancas"), síntomas para teniosis (defecación de proglótidos) y síntomas de cisticercosis (dolores de cabeza, desmayos y ataques epilépticos). Sin embargo, todos estos factores no fueron lo suficientemente relevantes como para lograr que se presentará $T$. solium en la población de vendedores de alimentos. 
La ausencia de $T$. solium en la población estudiada, coincide con los informes acerca de la frecuencia de teniosis en zonas urbanas y semiurbanas; como se informa en expendedores de alimentos de dos zonas de la Ciudad de México $^{26}$ y en soldados de un campo militar ubicado en la Ciudad de México ${ }^{27}$. En el primer trabajo, realizado en expendedores de alimentos ubicados en Ciudad Universitaria y en la colonia Santo Tomás, se encontró que de 70 muestras fecales una resultó positiva a la prueba de ELISA para coproantígeno y que las condiciones higiénico-sanitarias de los expendedores no eran favorables (factor que probablemente influyó en la baja frecuencia encontrada, como sucedió en este estudio). En el segundo trabajo, los autores encontraron una prevalencia del $0,1 \%$ para teniosis en estudio CPS y $0,5 \%$ por ELISA para coproantigeno; un factor fuertemente asociado que llama la atención fue que los individuos positivos refirieron comer carne de cerdo en comercios ambulantes. En el presente trabajo se encontró que el $92 \%$ de la población de vendedores de alimentos refirió comer carne de cerdo; y de estos, $21 \%$ mencionó consumirla diariamente; y además, $73 \%$ consumía la carne de cerdo que comerciaba en el local pero no se encontraron casos de seropositividad.

Para estudios ulteriores en poblaciones semejantes a la del presente informe es necesario aumentar el número de comerciantes y utilizar técnicas inmunológicas de mayor sensibilidad y especificidad, como el ELISA para coproantígeno para la búsqueda de teniasis. ${ }^{28} \mathrm{Se}$ recomienda a las autoridades dar educación sanitaria a los comerciantes y a los consumidores de alimentos, porque, a pesar de no encontrar teniosis, sí se identificaron otras parasitosis intestinales que afectan la salud de los comerciantes y de la población que consume sus productos.

\section{RESUMEN}

Con el objeto de conocer la prevalencia de teniosis y cisticercosis en comerciantes de alimentos de mercados de la Delegación política de Xochimilco de la Ciudad de México, se encuestaron 262 comerciantes de 11 mercados. En ellos se hicieron estudios coproparasitoscopicos seriados y en la sangre se buscaron anticuerpos específicos contra cisticerco mediante inmunoelectrotransferencia. Sólo 115 entregaron las muestras fecales, y de ellas, en 110 se obtuvo la muestra de sangre. La seroprevalencia de anticuerpos para cisticercos fue del $0,9 \%$. En ninguna muestra de material fecal se encontraron huevos de $T$. solium. Entre los comerciantes, $56,4 \%$ vendían algunos alimentos con carne de cerdo; $11,3 \%$ de ellos criaban los cerdos en su casa y los sacrificaban de manera clandestina para vender su carne. Por lo tanto, los comerciantes de alimentos con carne de cerdo, que expenden sus productos en estos mercados, parecen estar rara vez expuestos a la T. solium.

\section{REFERENCIAS}

1.- ANTONIUK S. Epidemiología de la neurocisticercosis. Rev Neurol 1999; 29: 331-4.

2.- ROMÁN G, SOTELO J, DEL BRUTO O et al. A proposal to declare neurocysticercosis an inter-national reportable disease. Bull World Health Organ 2000; 78: 399-406.

3.- SARTI E. La teniosis y cisticercosis por Taenia solium. Salud Pública Mex 1997; 39: 225-31.

4.- SARTI E, FLISSER A, CORREA D et al. Teniasis y cisticercosis. En: diez problemas relevantes de salud pública en México. México. Secretaria de Salud; 1999. p. 117-44.

5..- LARRALDE C, PADILLA A, HERNÁNDEZ M et al. Seroepidemiología de la cisticercosis en México. Salud Pública Mex 1992; 34: 197-210.

6.- DEL BRUTO O H. Neurocisticercosis. Rev Neurol 1999; 29: 456-66.

7.- CARPIO A, ESCOBAR A, HAUSER A. Cysticercosis and epilepsy: a critical rewiew. Epilepsia 1998; 39: 1025-40.

8.- WHITE A C. Neurocysticercosis: updates on epidemiology, pathogenesis, diagnosis, and management. Annu Rev Med 2000; 51: 187-206.

9.- SÁNCHEZ A L, ALLEBECK P, COSENZA H et al. Epidemiological study of Taenia solium infections in a rural village in Honduras. Ann Trop Med Parasitol 1997; 91: 163-71.

10.- CAO W, PLOEG C, XU J et al. Risk factors for human cisticercosis morbidity: a population based case-control study. Epidemiol Infect 1997; 119: 231-5.

11.- SÁNCHEZ A L, MEDINA M T, LJUNGSTRÖM I. Prevalence of taeniasis and cisticercosis in a population of urban residence in Honduras. Acta Trop 1998; 69: 141-9.

12.- RODRÍGUEZ R, FRASSER A, ALLAN J C et al. Epidemiological study of Taenia solium taaeniasis/ cysticercosis in a rural village in Yucatan state, Mexico. Ann Trop Med Parasitol 1999; 93: 57-67.

13.- SÁNCHEZ A L, LINDBÄCK J, SCHANTZ P et al. A population-based, case-control study of Taenia solium taeniasis and cysticercosis. Ann Trop Med Parasitol 1999; 93: 247-58.

14.- MARTÍNEZ J J, JARAMILLO C, ALUJA A et al. Epidemiología de la cisticercosis en cerdos de una 
comunidad rural del estado de Guerrero, México. Vet Méx 1997; 28: 281-6.

15.- GRUPO DE TRABAJO SOBRE CISTICERCOSIS EN EL PERÚ. La comercialización de cerdos cisticercóticos en la sierra del Perú. Bol Oficina Sanit Panam 1994; 116: 427-34.

16.- CUADERNO ESTADÍSTICO DELEGACIONAL. Xochimilco. México: Instituto Nacional de Estadística Geográfica e Informática (INEGI); 1997. p 3-131.

17.- ANUARIO ESTADÍSTICO DEL DISTRITO FEDERAL. MÉXICO: Instituto Nacional de Estadística Geográfica e Informática (INEGI); 1996. p 216-9.

18.- RITCHIE L S. An ether sedimentation technique for routine stool examinations. Bull US Army Med Dept 1948; 8: 326

19.- TSANG V, BRAND J, BOYER A. An enzyme-linked immunoelectrotransfer blot assay and glycoprotein antigens for diagnosing human cysticercosis (Taenia solium). J Infect Dis 1989; 159: 50-9.

20.- BOGITSH B, CHENG T. Human parasitology. 2a ed. New York: Academic Press; 1998.

21.- SALAZAR P M, DE HARO I, RUIZ A et al. Investigación de otro probable mecanismo de infección en la cisticercosis. I Informe de los hallazgos preliminares. Arch Invest Méd Méx 1984; 15: 205-13.

22.- ACEVEDO H A. Economic impact of porcine cysticercosis. En: Flisser A, Willms K, Laclette P, et al. Cysticercosis. Present state of knowledge and perspectives. Ed Academic Press, New York, 1982: 63-7.
23.- ARÁMBULO P, ALMEIDA C, CUÉLLAR J et al. La venta de alimentos en la vía pública en América Latina. Bol Oficina Sanit Panam 1995; 118: 97-107.

24.- MOORE A, LUTWICK L, SCHANTZ P et al. Seroprevalence of cysticercosis in an orthodox jewish community. Am J Trop Med Hyg 1995; 53: 439-42.

25.- GARCÍA H, ARAOZ R, GILMAN R et al. Increased prevalence of cysticercosis and taeniasis among professional fried pork vendors and general population of a village in the peruvian highlands. Am J Trop Med Hyg 1998; 59: 902-5.

26.- SÁNCHEZ A P, AMBROSIO J, AVILA G et al. Frecuencia de teniosis y cisticercosis en expendedores de alimentos. Rev Fac Med UNAM 2002; 45: 60-3.

27.- GARCÍA M L, TORRES M, CORREA D et al. Prevalence and risk of cysticercosis and taeniasis in an urban population of soldiers and their relatives. Am J Trop Med Hyg 1999; 61: 386-9.

28.- MORÁN C, CRUZ V. Teniosis-cisticercosis. Epidemiología y factores de riesgo. Rev Fac Med UNAM 2000; 43: 67.

Agradecimientos: Los autores agradecen a la Dra. Mirella Loustalot Directora de los Servicios de Salud Pública del D.F., así como al personal que laboran en los Centros de Salud de la Secretaria de Salud de la Delegación Xochimilco por su valiosa cooperación. También agradecemos a la Dra. Guadalupe García y a la QFB. Mónica Mirabal la revisión critica del manuscrito.

\section{ANUNCIO}

\section{PARASITOLOGIA LATINOAMERICANA EN INTERNET}

Información sobre Parasitología Latinoamericana y su versión electrónica se puede ahora encontrar en Internet. Dirección: www.scielo.cl 